\title{
DNA methylome profiling of circulating tumor cells in lung cancer at single base-pair resolution
}

\author{
Lei Zhao ${ }^{1,2,3} \cdot$ Xiaohong Wu $\mathbb{1}^{4} \cdot$ Junnian Zheng $\mathbb{1}^{1,2} \cdot$ Dong Dong $\mathbb{D}^{1,2}$
}

Received: 6 October 2020 / Revised: 14 December 2020 / Accepted: 13 January 2021 / Published online: 9 February 2021

(c) The Author(s), under exclusive licence to Springer Nature Limited 2021. This article is published with open access

\begin{abstract}
DNA methylation plays a pivotal role in regulating cellular processes, and altered DNA methylation pattern is a general hallmark of cancer. However, DNA methylome in circulating tumor cells (CTCs) is still a mystery due to the lack of proper analytical techniques. We introduced an efficient workflow, LCM- $\mu$ WGBS, which can efficiently profile the DNA methylation of microdissected CTC samples. LCM- $\mu$ WGBS combines the laser capture microdissection (LCM)-based CTC capture method and whole-genome bisulfite sequencing in very small CTC population ( $\mu$ WGBS) to gain insight into the DNA methylation landscape of CTCs. We herein profiled the DNA methylome of CTCs from lung cancer patients. Deriving from a comprehensive analysis of CTC methylome, a unique "CTC DNA methylation signature" that is distinct from primary lung cancer tissues was identified. Further analysis showed that promoter hypermethylation of epithelial genes is a hallmark of stable epithelial-mesenchymal transition process. Moreover, it has been suggested that CTCs are endowed with a stemness-related feature during dissemination and metastasis. This work constitutes a unique DNA methylation analysis of CTCs at single base-pair resolution, which might facilitate to propose noninvasive CTC DNA methylation biomarkers contributing to clinical diagnosis.
\end{abstract}

\section{Introduction}

Circulating tumor cells (CTCs) are cells that have shed into the bloodstream from primary or metastatic tumors and

These authors contributed equally: Lei Zhao, Xiaohong Wu

Supplementary information The online version contains supplementary material available at https://doi.org/10.1038/s41388021-01657-0.

Junnian Zheng

jnzheng@xzhmu.edu.cn

$\triangle$ Dong Dong

ddong@xzhmu.edu.cn

1 Cancer Institute, Xuzhou Medical University, Xuzhou, Jiangsu, China

2 Center of Clinical Oncology, Affiliated Hospital of Xuzhou Medical University, Xuzhou, China

3 Shanghai Key Laboratory of Regulatory Biology, Institute of Biomedical Sciences, School of Life Sciences, East China Normal University, Shanghai, China

4 Department of General Surgery, Affiliated Yixing Hospital of Jiangsu University, Yixing, 214200 Jiangsu, China circulate in the bloodstream of cancer patients. CTCs have the potential to form metastases in distant organs that are ultimately responsible for the vast majority of cancer-related deaths [1]. The development of "liquid biopsies" presents new opportunities for noninvasive monitoring of cancer, with applications ranging from early detection to treatment selection and monitoring response. Given the critical role of CTCs in the metastatic cascade, the number of CTCs in patient peripheral blood has been found to correlate with the diagnosis and clinical outcomes. The enumeration of CTCs enables detecting cancers and monitoring therapeutic response by a noninvasive way. Moreover, CTCs captured as a "liquid biopsy" contain intact genomic and transcriptomic information of cancer cells, which made them suitable for use as clinical biomarkers [2-5]. The rarity of CTCs in circulating blood becomes the primary issue to overcome for CTC assay. Differentiating CTCs from the vast contaminating leukocytes background requires highly sensitive and specific assays. Classical CTC enumeration includes the presence of cell surface epithelial cell adhesion molecule (EpCAM), cytoplasmic epithelial cytokeratins, and the absence of the hematopoietic CD45 marker [6]. However, recent works have documented that CTCs were frequently in an intermediate epithelial-to-mesenchymal 
(EMT) state, and some epithelial markers become rapidly downregulated when cancer cells enter the bloodstream [7-9]. To date, the primary method for isolating and capturing CTCs is micromanipulation; however, this process is too time consuming and laborious for routine clinical application. Laser capture microdissection (LCM) has been widely used to isolate cells from solid tissue samples, especially in fixed in formalin and embedded in paraffin samples. LCM enables a highly accurate isolation of CTCs from leukocytes, and has been successfully used to isolate CTC single cells. The ability of LCM to capture CTCs without loss of cells and cell integrity is critical in subsequent sequencing analysis [10].

DNA methylation is an epigenetic mechanism used by cells to control gene expression, which can fix genes in the "off" position [11, 12]. Extensive DNA methylation perturbation has been widely explored in human cancer, causing changes in gene regulation that promote oncogenesis. Understanding epigenetic changes shows promise for improving the characterization of malignancy to predict diagnosis and prognosis [13-16]. Some DNA methylation changes are even found in some cases of a specific type of cancer. With the emerging of high-throughput technologies, such as comprehensive DNA methylation microarrays and genome-wide bisulfite sequencing (WGBS), large amount of DNA methylation profiling data have been generated $[12,17]$. These data provided us great importance to improve the prediction of cancer diagnosis and prognosis. To date, few works focused on the DNA methylation analysis of CTCs, which is most likely explained by the combined technical challenges of CTC isolation and DNA methylation analyses on extremely rare cells. Some pioneering studies explored the DNA methylation on some specific genes. For example, Chimonidou et al. investigated the methylation status of three tumor-associated genes (CST6, BRMS1, and SOX17) in breast cancer CTCs [18-20], and the results suggested that the expressions of these genes are modulated by DNA methylation.

The epigenetic landscape of CTCs remains a largely unexplored field with great potential. Considering the mounting evidence for the role in epigenetics, especially DNA methylation in several cellular mechanisms, elucidation of DNA methylation profiles of CTCs is essential to understand the molecular mechanism of tumor metastasis $[21,22]$. In this study, we sought to better understand the whole-genome DNA methylome of CTCs at single basepair resolution. LCM platform was employed for CTCs isolation. CTC DNA methylation data were generated by using WGBS method from lung cancer CTCs. Our work comprehensively addressed the epigenetic state of CTCs, and provided an epigenetic picture to elucidate mechanisms during dissemination and metastasis and to develop tumor biomarkers.
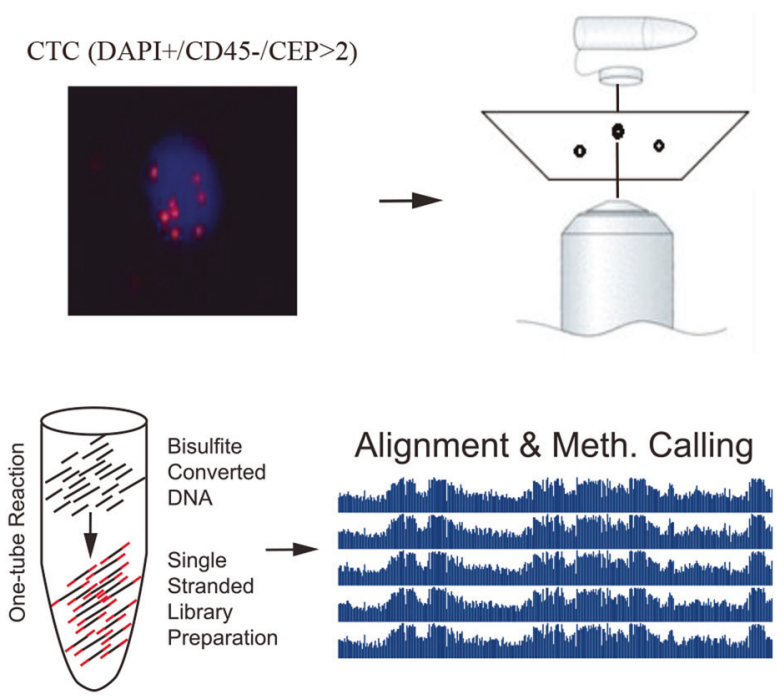

Fig. 1 Schematics of LCM- $\mu$ WGBS for CTC DNA methylation profiling. Subtraction enrichment and immunostaining-fluorescence in situ hybridization (SE-iFISH) was used to detect CTCs. LCM method was then applied to capture CTCs. At last, whole-genome bisulfite sequencing of a small number of cells was employed for DNA methylation profiling.

\section{Results}

\section{LCM- $\mu$ WGBS workflow for DNA methylation profiling}

Subtraction enrichment and immunostaining-fluorescence in situ hybridization (SE-iFISH) was an established method to detect CTCs. In general, CTCs were characterized as nucleated cells with epithelial markers and/or hyperdiploid, with absence of lymphocytic marker CD45. This method is independent of downregulation or loss of EpCAM expression. According to previous works [23, 24], CTCs were defined as DAPI+/CD45-/CEP8 $>2$ in our work (Fig. 1). LCM method was then applied to capture CTCs on the polyethylene-napthalate (PEN) membrane-coated slide. The enriched CTC samples were encapsulated in a hydrogel matrix, which made them easily to be isolated by LCM and compatible with downstream analysis. $\mu \mathrm{WGBS}$ is an established DNA methylation analysis method of a small number of cells based on the post-bisulfite adapter tagging (PBAT) assay. With the attempt to robustly and efficiently identify the whole-genome DNA methylation profiles of CTCs, we have developed a LCM- $\mu$ WGBS workflow by combining LCM-based CTC capture and $\mu$ WGBS method (Fig. 1).

By optimizing multiple steps in the procedure, we can now acquire high-quality DNA methylation data down to LCM dissected cells. To technically validate this strategy, we detected the DNA methylome measured from the introduction of individually micromanipulated cells from 
Table 1 Statistics of $\mu$ WGBS data of CTCs.

\begin{tabular}{lllllllll}
\hline Sample ID & Origins & CTC\# & $\begin{array}{l}\text { Total } \\
\text { clean reads }\end{array}$ & $\begin{array}{l}\text { Percentage of } \\
\text { mapped reads }\end{array}$ & Coverage & Unique CpGs & $\begin{array}{l}\text { CpG } \\
\text { coverage }\end{array}$ & $\begin{array}{l}\text { Bisulfite } \\
\text { conversion rate }\end{array}$ \\
\hline Patient1_CTC & No LCLM & 16 & 255320380 & $38 \%$ & 0.544 & 18602441 & 0.648 & 0.992 \\
Patient2_CTC & No LCLM & 11 & 131833442 & $47 \%$ & 0.189 & 6262136 & 0.218 & 0.991 \\
Patient3_CTC & No LCLM & 13 & 131827972 & $50 \%$ & 0.314 & 10285461 & 0.358 & 0.990 \\
Patient4_CTC & No LCLM & 10 & 131916942 & $49 \%$ & 0.182 & 5734767 & 0.200 & 0.984 \\
Patient5_CTC & No LCLM & 18 & 131575810 & $50 \%$ & 0.108 & 3532767 & 0.123 & 0.992 \\
Patient6_CTC & LCLM & 18 & 263668458 & $48 \%$ & 0.512 & 17650885 & 0.615 & 0.991 \\
Patient7_CTC & LCLM & 22 & 176002976 & $33 \%$ & 0.639 & 19175555 & 0.668 & 0.995 \\
Patient8_CTC & LCLM & 18 & 163784488 & $37 \%$ & 0.572 & 17405709 & 0.606 & 0.995 \\
Patient9_CTC & LCLM & 20 & 141161092 & $25 \%$ & 0.545 & 16200091 & 0.564 & 0.994 \\
Patient10_CTC & LCLM & 20 & 141161092 & $50 \%$ & 0.641 & 19896348 & 0.693 & 0.995 \\
Patient11_CTC & No LCLM & 11 & 133144320 & $37 \%$ & 0.148 & 4550251 & 0.159 & 0.992 \\
Patient12_CTC & No LCLM & 16 & 130581964 & $47 \%$ & 0.210 & 8219373 & 0.286 & 0.991 \\
Patient13_CTC & No LCLM & 18 & 131534266 & $50 \%$ & 0.151 & 4884290 & 0.170 & 0.992 \\
Patient14_CTC & No LCLM & 18 & 136890898 & $51 \%$ & 0.234 & 7522107 & 0.262 & 0.990 \\
Patient15_CTC & No LCLM & 16 & 131982568 & $48 \%$ & 0.189 & 6222565 & 0.217 & 0.992 \\
\hline
\end{tabular}

LCLM lung cancer liver metastasis.

lung cancer cell line A549 into $5 \mathrm{ml}$ of blood from a healthy donor, followed by cancer cells subtraction enrichment and LCM- $\mu$ WGBS processing. LCM procedure requires a dedicated instrument in a confined space to avoid contamination of experimental materials. The parameters for LCM should be set at the lowest energy to ensure the integrity of those dissected cells. Because of the rarity of CTCs, experimental groups of 10 and 50 A549 cells were isolated from the mixed blood cells using LCM method, respectively. Those harvested cells of interests were pooled together for each sample to provide enough starting materials for subsequent $\mu$ WGBS. The result showed that the conversion rate resulting from bisulfite treatment is more than $98 \%$ for the 10-cell and 50-cell A549 DNA methylome, suggesting the high fidelity of this method. An average of 145 million paired-end reads were generated, covering $58 \%$ of the human genome (Table 1). We determined the presence of $16,660,129$ and 19,437,132 CpG sites for the 10-cell and 50-cell A549 DNA methylome, which account for $58 \%$ and $61 \%$ of the total $\mathrm{CpG}$ sites at the whole-genome-wide level. To evaluate the performance of LCM- $\mu$ WGBS, we benchmarked them against human A549 cell line WGBS data. The result showed significantly high Pearson correlation coefficients of 0.82 and 0.89 with 10-cell A549 and 50-cell A549, respectively (Fig. 2). As for the gene promoter regions, we observed Pearson correlation coefficients of 0.91 and 0.94 with 10-cell A549 and 50-cell A549 (Fig. 2), respectively. Moreover, these two data display the similar DNA methylation level as the A549 cell line WGBS data (Supplementary Fig. S1). These results suggested that LCM- $\mu$ WGBS method provides an accurate and efficient way to interrogate CTCs DNA methylation information at the whole-genome-wide level.

\section{DNA methylation signature of lung cancer CTCs}

According to the CTCs collection strategy, CTCs were isolated from a cohort of 15 lung cancer patients in this way, yielding between 10 and $22 \mathrm{CTCs} / 5 \mathrm{ml}$ of patient blood (Table 1). To gain a comprehensive insight into the DNA methylation changes during CTC dissemination and metastasis, genome-wide DNA methylation profiling of 15 CTC samples, 5 matched primary tumor samples, and 5 matched adjacent normal tissue samples derived from lung cancer patients were performed using LCM- $\mu$ WGBS method (Fig. 3a, Table 1, Supplementary Table S1). Biopsy specimens contain a mix of tumor and normal tissue cells, tumor infiltrating lymphocytes. The tumor tissues had been stained with H\&E before $\mu$ WGBS. Moreover, we employed a deconvolution method to estimate the tumor purity [25]. The result showed that the tumor purity was $>90 \%$. Bisulfite sequencing reads were generated with an average of 150 million reads per sample $(7.5 \times$ coverage). Two CTC samples (patient 1 and patient 6) were sequenced to 250 million reads per sample $(12.5 \times$ coverage), and sequencing saturation analysis was performed to determine the sequencing coverage needed for LCM- $\mu$ WGBS. As sequencing depth increases, more $\mathrm{CpG}$ sites were detected. The number of $\mathrm{CpG}$ sites reaches saturation at the $6 \times$ to $10 \times$ genome coverage (Supplementary Fig. S2). This result suggested that the sequencing depth of this work is enough for further analysis. After mapping to the human reference genome 
Fig. 2 The repeatability and robustness of $\mathrm{LCM}-\mu \mathrm{WGBS}$ workflow. Micromanipulated A549 cells were spiked into the whole blood of the healthy donors. 10 cells and 50 cells were captured from the mixed samples, respectively, and the DNA methylomes were profiled subsequently. a DNA methylation levels of A549 cell lines measured using LCM$\mu$ WGBS and WGBS at the whole-genome-wide level. b DNA methylation levels of A549 cell lines measured using LCM- $\mu$ WGBS and WGBS at the promoter region. a)
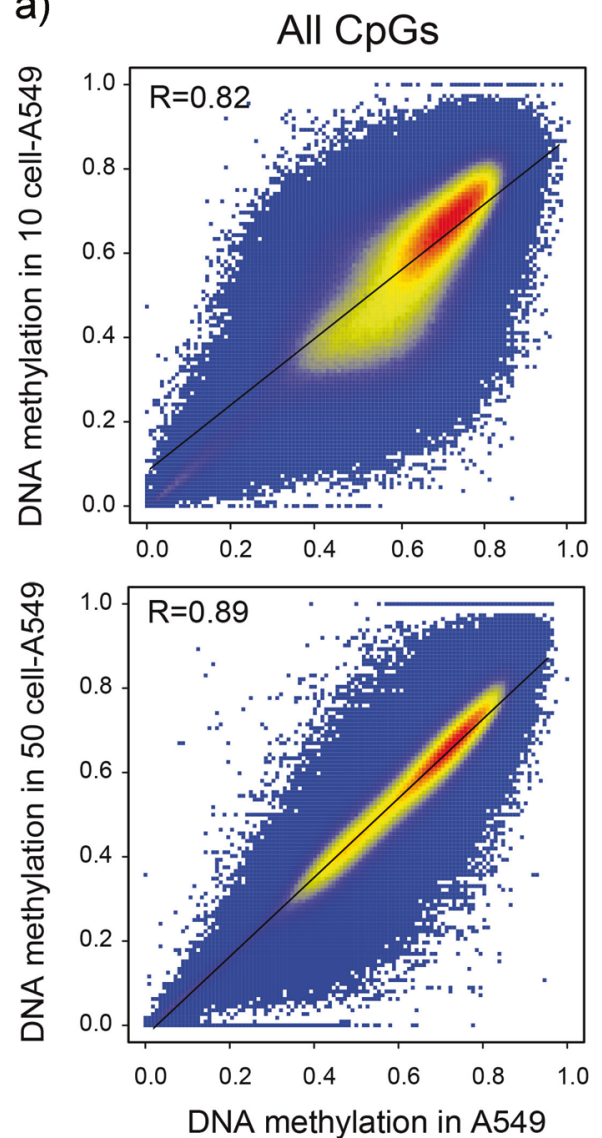

b)
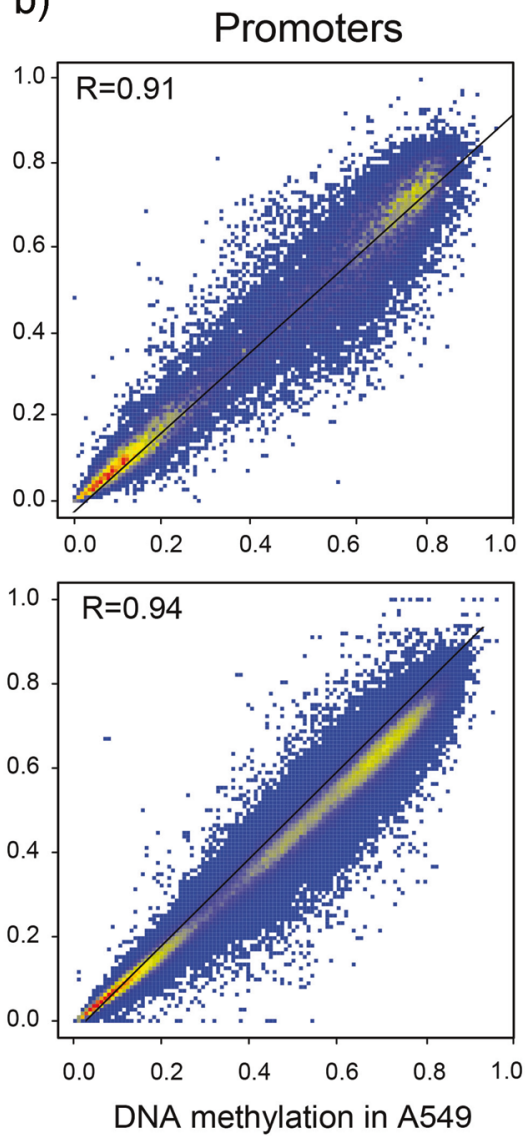

(hg38), we identified an average of 13,474,616 CpG sites for CTC samples, which covered on average $46.9 \%$ of the total $\mathrm{CpG}$ sites at the genome-wide level. We investigated the DNA methylation levels along all chromosomes by assessing with a 300-kb sliding window. To assess the DNA methylation similarities of the CTCs samples, Pearson correlation coefficients were calculated for every two samples. The average correlation coefficient was observed to be 0.68 , indicating a relatively higher consistency of CTCs samples. Further unsupervised hierarchical clustering analysis showed that CTCs clustered together as a single group, indicating that CTCs resemble each other more closely than their primary tumor counterparts (Fig. 3b).

Consistent with previous reports [12,17], normal tissues have higher CpG methylation level (70-80\%), whereas dramatic DNA methylation losses occur in cancer samples in this context. Notably, we found that global DNA methylation was significantly lower in CTCs compared with normal and cancer samples (Wilcoxon rank sum test, $P<2.2 \times 10^{-16}$, Fig. 3c). This progressive decrease in global DNA methylation from normal tissues to primary tumors and, in turn, to their associated CTCs suggested a successive loss of DNA methylation during tumorigenesis.
Further analysis suggested a significant loss of the association between methylation levels of adjacent $\mathrm{CpG}$ sites (Fig. 3d), indicating DNA methylation loss may occur randomly rather than at consecutive $\mathrm{CpG}$ sites. From a genomic perspective, the decrease of DNA methylation occurred at all genomic compartments, such as promoter, gene body, intron, and intergenic region (Fig. 3e). After we subgrouped the promoters based on their $\mathrm{CpG}$ contents, we found that the CTC DNA exhibited more unmethylated CpGs at the CpG-poor promoters (Wilcoxon rank sum test, $P<2.2 \times 10^{-16}$ ) and similar methylated $\mathrm{CpGs}$ at $\mathrm{CpG}$ island promoters (Wilcoxon rank sum test, $P=0.37$, Fig. 3 e) than the primary tumor samples, suggesting hypomethylated $\mathrm{CpG}$ sites in CTCs mainly occurred in the promoter regions lacking $\mathrm{CpG}$ islands.

\section{Identification of differentially methylated regions (DMRs)}

The discrepancies between the CTCs and primary lung cancer DNA methylome prompted us to detect DMRs. DMRs were identified using a smoothing approach, and $\mathrm{CpGs}$ with correlated methylation values were grouped together (see "Materials and methods"). We totally 
Fig. 3 Genome-wide DNA methylation changes in lung cancer CTCs. a Whole-genome representation of DNA methylation levels of lung cancer CTCs, primary tumors and normal tissues. $\mathbf{b}$ Unsupervised hierarchical clustering of DNA methylation data from lung cancer CTCs, primary tumors and normal tissues. c DNA methylation level was significantly lower in CTCs compared with normal and cancer samples.

d Correlation of DNA methylation levels between neighboring $\mathrm{CpG}$ sites. e DNA methylation levels among different genomic sequences. The error bars represent the $95 \%$ confidence intervals. a)

b)

c)
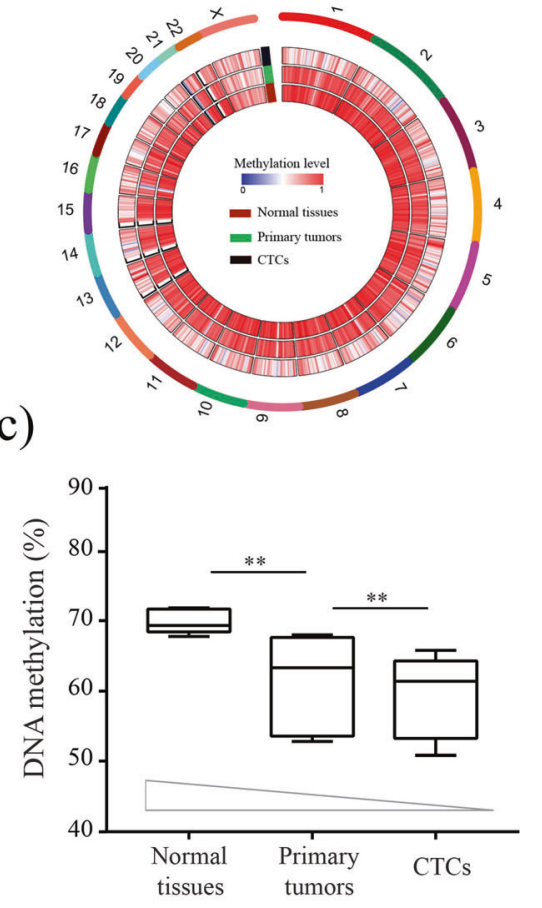

d)

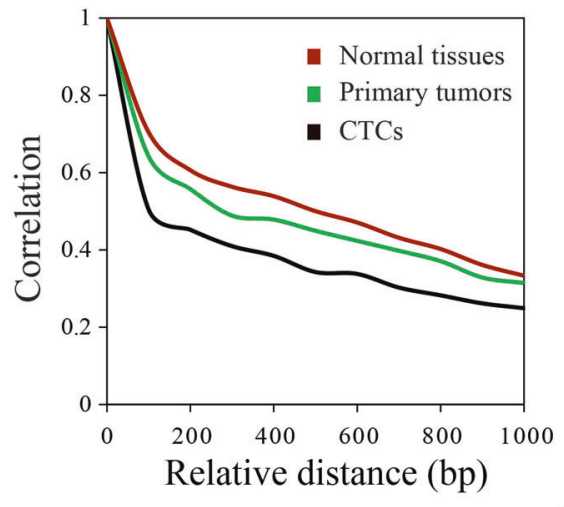

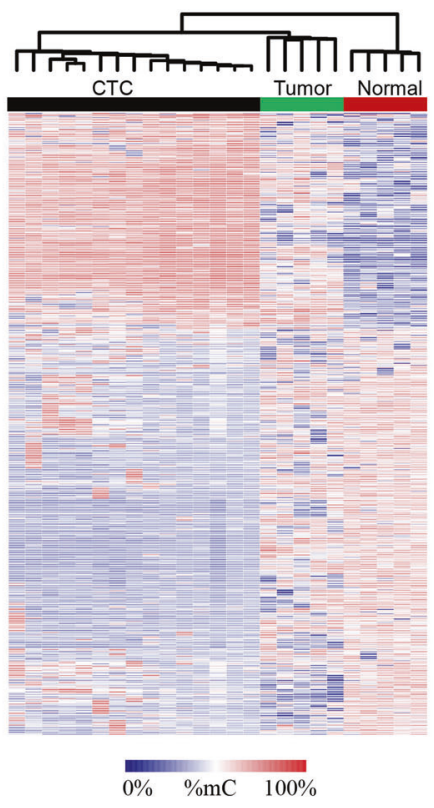

e)

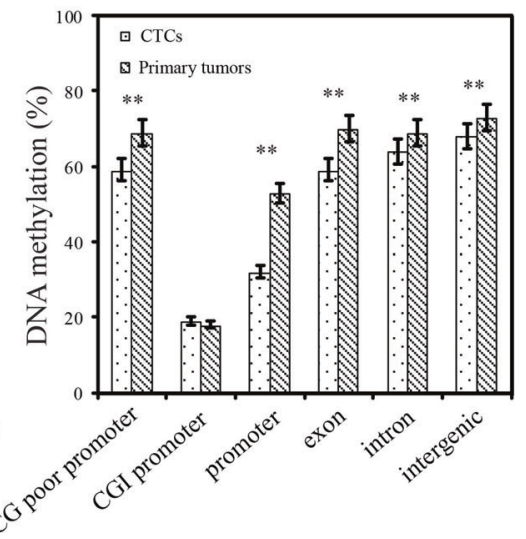

identified 64,607 DMRs (ctc-DMRs) between CTCs and primary tumor samples, and 28,414 DMRs (t-DMRs) between primary tumors and normal tissues. A total of 3292 DMRs were classified as overlapping (Fig. 4a). The complete lists of ctc-DMRs and t-DMRs can be found in Supplementary Tables S2 and S3. The ctc-DMRs and t-DMRs represent 2,223,957 CpG sites and 1,281,976 CpG sites in the reference genome that are distributed across all human chromosomes, respectively. To validate those identified DMRs, we performed a pairwise sample comparison, and the result showed that most of the DMRs $(90 \%)$ were existed in at least three samples (Supplementary Fig. S3).

The DMRs were distributed across the human genome, and provided information on all genomic contexts. DMRs were identified in promoters $(16,719$ for ctc-DMRs and 6368 for t-DMRs), exonic, $(22,901$ for ctc-DMRs and 10,345 for t-DMRs), intronic (39,857 for ctc-DMRs and 16,824 for t-DMRs), and intergenic regions (35,140 for ctcDMRs and 15,826 for t-DMRs). Consistent with previous reports, $1884(6.6 \%)$ of these t-DMRs were hypermethylated, whereas 26,530 (93.4\%) were hypomethylated. Global DNA hypomethylation is a common genetic feature of lung cancer tumorigenesis. As for ctc-DMRs, 43,283 $(67.0 \%)$ of them were hypomethylated, indicating a greater tendency toward decreased DNA methylation at aberrantly methylated loci in CTCs (Fig. 4b).

Hypomethylated regions in CTC samples covered $4.2 \%$ of the entire human genome. In all, $42.8 \%$ of the hypomethylated regions are located within gene promoters. Previous reports suggested that DNA hypomethylation and 
a)

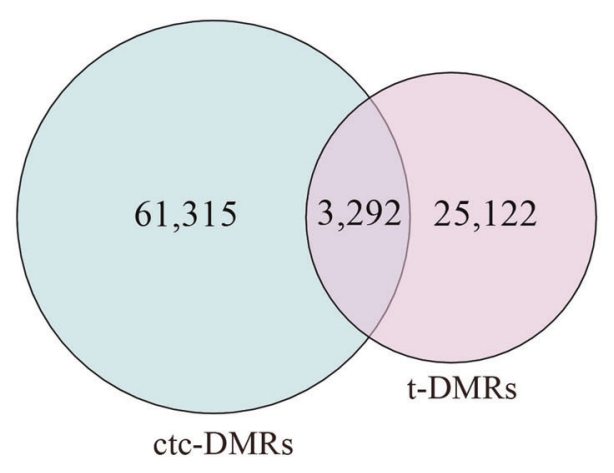

c)

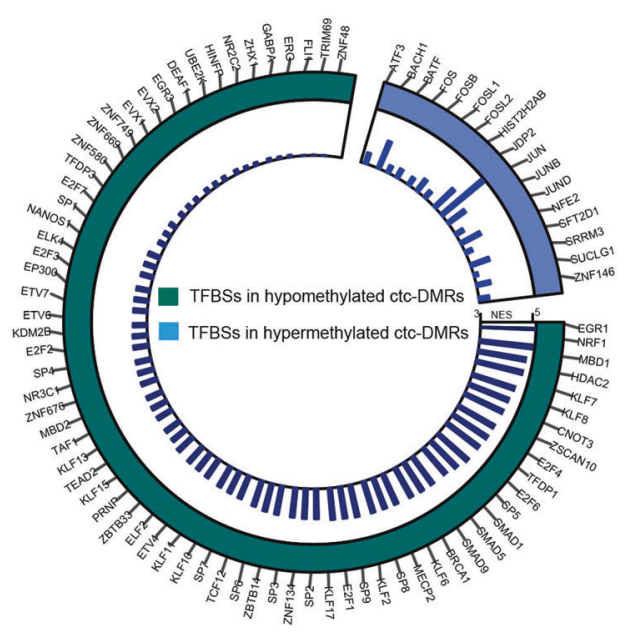

b)

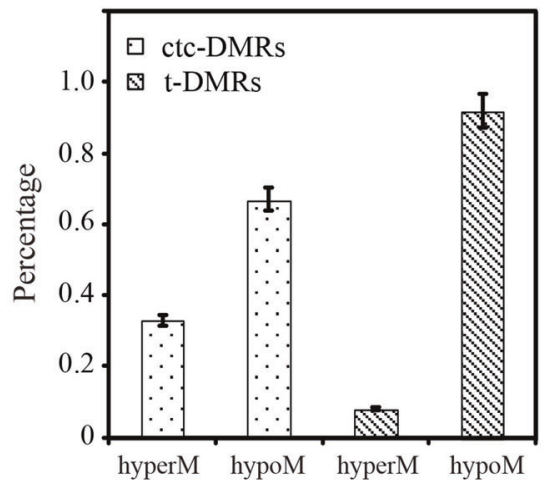

d)

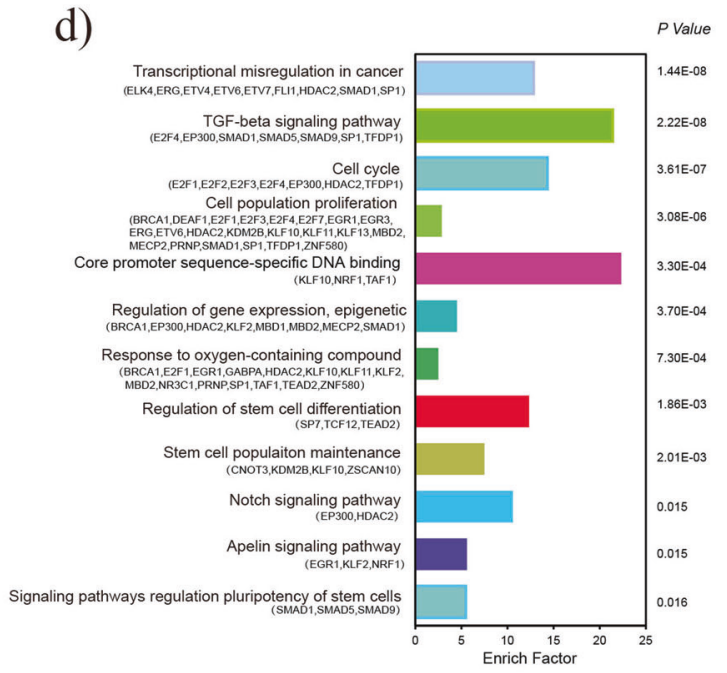

e)

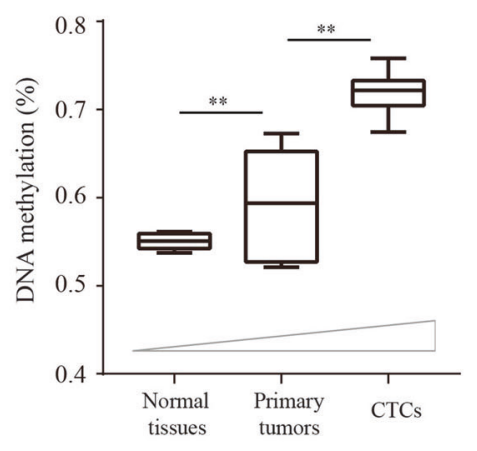

f)

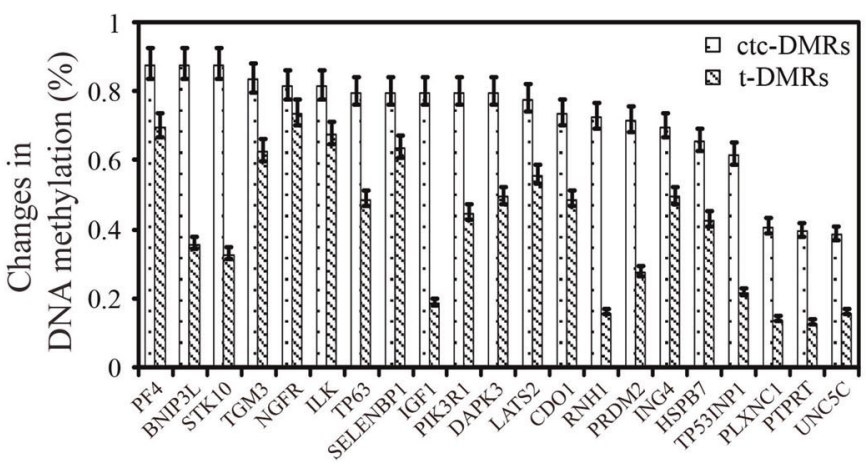

Fig. 4 Functional characterization of DMRs. a The number of DMRs between CTCs and primary tumors (ctc-DMRs), and the number of DMRs between primary tumors and normal tissues ( $t$ DMRs). A total of 3292 DMRs were classified as overlapping DMRs. b More hypomethylated DMRs were observed than hypermethylated DMRs in both ctc-DMRs and t-DMRs. c Normalized enrichment score (NES) representing enrichment (NES $\geq 3.4$ ) of transcription factor binding sites (TFBSs) in hypomethylated ctc-DMRs and hypermethylated ctc-DMRs. d Integrated pathway analysis of TFBSs in hypomethylated ctc-DMRs. The bars represent the percentage of genes detected per pathway category with $P$ value $\leq 0.05$. e CpG sites in hypermethylated t-DMRs further gained methylation intensities in CTCs. f Representative tumor suppressor genes show increased methylation in CTCs and primary tumors. The error bars represent the 95\% confidence intervals. 
Table 2 KEGG pathway enrichment analysis.

\begin{tabular}{lllll}
\hline & KEGG ID & Description & No. of genes & F.D.R. \\
\hline Hypomethylation & hsa04392 & Hippo signaling pathway & 14 & 0.03793 \\
& hsa04012 & ErbB signaling pathway & 41 & 0.00192 \\
& hsa04120 & Ubiquitin mediated proteolysis & 61 & 0.00158 \\
& hsa04350 & TGF-beta signaling pathway & 36 & 0.02109 \\
& hsa01521 & EGFR tyrosine kinase inhibitor resistance & 34 & 0.02774 \\
& hsa04668 & TNF signaling pathway & 44 & 0.03742 \\
& hsa04015 & Rap1 signaling pathway & 79 & 0.04836 \\
& hsa04152 & AMPK signaling pathway & 47 & 0.06318 \\
& hsa04340 & Hedgehog signaling pathway & 20 & 0.07179 \\
hsa00510 & N-Glycan biosynthesis & 21 & 0.07652 \\
hsa04066 & HIF-1 signaling pathway & 39 & 0.07821 \\
hsa04810 & Regulation of actin cytoskeleton & 34 & 0.00002 \\
hsa04530 & Tight junction & 16 & 0.02035 \\
hsa04974 & Protein digestion and absorption & 16 & 0.00087 \\
hsa04020 & Calcium signaling pathway & 26 & 0.00092 \\
hsa04370 & VEGF signaling pathway & 12 & 0.00125 \\
hsa04510 & Focal adhesion & 27 & 0.00192 \\
hsa04670 & Leukocyte transendothelial migration & 17 & 0.00370 \\
hsa04015 & Rap1 signaling pathway & 26 & 0.00746 \\
hsa04973 & Carbohydrate digestion and absorption & 8 & 0.01561 \\
hsa04520 & Adherens junction & 11 & 0.01809 \\
hsa04014 & Ras signaling pathway & 26 & 0.02038 \\
hsa04964 & Proximal tubule bicarbonate reclamation & 5 & 0.02578 \\
\hline
\end{tabular}

transcription factor binding sites (TFBSs) are highly correlated, describing a codependency of these regulatory mechanisms. In this work, we found that CTC DNA hypomethylation and transcription factor occupancy revealed a significant relationship (Fisher's exact test, $P<$ $\left.2.2 \times 10^{-16}\right)$. We then analyzed ctc-DMRs using i-cisTarget method [26], and found a significant enrichment for several TFBSs among DNA hypomethylated regions that are specific to CTCs (Fig. 4c). For example, transcription factor EGR1 shows the most remarkable enrichment in the hypomethylation regions specific to CTCs. EGR1 has been reported to be involved in the regulation of cell growth, differentiation, and apoptosis in several cancer types [27]. Transcription factors E2F1 and TCF12 both regulated target genes mainly involved in cell cycle and/or cell division processes [28, 29]. Further Gene Ontology analysis of global CTC hypomethylated TFBSs demonstrating that stemness-related transcription factors are significantly enriched to coordinately regulate proliferation and pluripotency (Fig. 4d), such as CNOT3, KDM2B, and KLF10. Thus, CTCs are clearly distinguishable from primary tumors based on their DNA methylation status at DMRs, where they feature more hypomethylated TFBSs.

Despite the progressive loss of DNA methylation during tumorigenesis, we found that $\mathrm{CpG}$ sites in the hypermethylated t-DMRs further gained methylation intensities in CTCs (Fig. 4e). Several tumor suppressor genes promoters that were hypermethylated in tumors showed a greater tendency toward increased methylation in CTCs with potential cancer-driving effects (Fig. 4f). For example, PIK3Rl is a suppressor of the mitogenic AKT pathway, and highly frequent promoter methylation and $P I K 3 R I$ has been reported in lung cancer. The promoter hypermethylation of $P I K 3 R I$ is significantly associated with gene expression in lung cancer samples (TCGA, LUAD, $r=0.54, P<2.2 \times 10^{-16}$ ). This focal gain of DNA methylation in tumors and even CTCs highlights the extensive changes in DNA methylation that occur in cancer progression and dissemination. To further gain insight in to the functional significance of DNA methylation changes, we performed KEGG pathway enrichment analysis for those hypo- and hypermethylation related genes (Table 2). This analysis revealed that genes in the hypomethylation regions are involved in the "TGFbeta signaling pathway," "hippo signaling pathway," and "hedgehog signaling pathway." These pathways have been documented to be significantly related to EMT process [30]. On the other extreme, genes in the hypermethylation regions are involved in the "tight junction," "focal adhesion" functions. 
Among ten lung cancer patients, only four of them had clinically detectable liver metastasis (LCLM, Table 1). We next sought to detect distinct patterns of liver metastatic CTCs. The result showed that global DNA methylation was nearly similar in CTCs derived from four patients diagnosed with LCLM compared with no LCLM group (Wilcoxon rank sum test, $P=0.36$, Supplementary Fig. S4). We totally detected 487 genes which showed significantly distinct DNA methylation in their promoter regions. Subsequently pathway analysis revealed higher activation of some known pathways in LCLM patients (Supplementary Table S4), such as Notch signaling pathway, Hippo signaling pathway, etc. The comparison of patient cohort of LCLM and no LCLM revealed the epigenetic signature of liver metastatic CTCs.

\section{CTC DNA methylation of epithelial, mesenchymal, and stem cell markers}

Previous works have documented that CTCs were frequently in an intermediate EMT state, and significantly lost the epithelial markers. Since different epithelial and mesenchymal genes are actually known to act in concert during EMT process, we herein comprehensively interrogated the epigenetic status of epithelial and mesenchymal markers on CTCs. As shown in Fig. 5, epithelial gene promoters are hypermethylated in CTC samples. For example, epithelial gene $C d h 1$ and EpCAM, key features of EMT process, were significantly hypermethylated in CTCs (Fig. 5). This result is consistent with previous findings that CTCs loss some of their epithelial characteristics, and promoter hypermethylation of epithelial

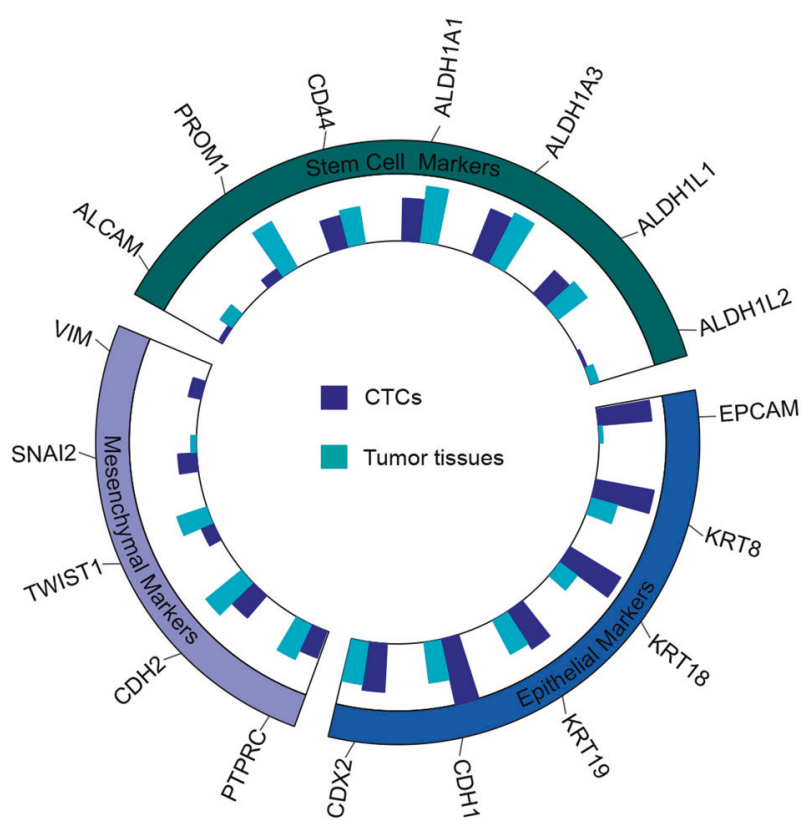

Fig. 5 Targeted analysis of DNA methylome data. DNA methylation levels of epithelial, mesenchymal and stem cell markers. DNA methylation levels of epithelial, mesenchymal, and stem cell markers. genes is hallmarks of stable EMT process. Furthermore, the DNA methylation of mesenchymal gene promoters are mixed, with some showing DNA hypermethylation in CTCs (Vim and Snail2) and others with DNA hypomethylation status in CTCs (TWIST1, CDH2, and PTPRC). The promoters of putative stem cell genes are hypomethylated in CTCs (such as $A L D H 1 A 1, C D 44$ ), which strongly supports the findings proposed with the TFBS enrichment analysis, suggesting that CTCs are endowed with a stemness-related feature, and this program may play a pivotal role in determining the metastasis-seeding ability of CTCs.

\section{Discussion}

The concept of liquid biopsies for noninvasive detecting and monitoring cancer has been embedded in our minds as a promising approach in cancer diagnosis and prognosis. CTCs provide a source of intact genomic and epigenomic information for lineage-based analysis [31]. Efficient isolation of CTCs in the bloodstream, followed by genomic and epigenomic quantification, may provide a highly specific diagnostic assay. Here, we have described a new workflow, LCM- $\mu$ WGBS, which can accurately and reproducibly profile the DNA methylome based on microdissected CTC samples at the single base-pair resolution. Our approach combines the CTC negative enrichment with LCM method, enabling isolation of CTCs with intact DNA, together with DNA methylation profiling in very small cell population. This work provided a comprehensive genome-wide analysis of the DNA methylation events that characterize CTCs in lung cancer, and compared the methylation changes with matched primary tumors and normal tissues. We herein have reinforced the notion of epigenetic disruption as a signature during dissemination and metastasis.

As expected, the DNA methylation level was significantly lower in primary lung cancer samples compared with normal tissues [12, 14, 17, 32]. We described a clear distinction in DNA methylation patterns between CTCs and primary tumors, mostly in the form of hypomethylation in the former. From a functional genomic standpoint, the DNA methylation decrease was observed in the CTC DNA covered all genomic compartments. Since DNA methylation changes were associated with profound effects on gene expression, it suggested that the progressive decrease in global DNA methylation plays important roles during tumorigenesis and blood-borne dissemination. Interestingly, we found progressive increase in DNA methylation in focal regions, suggesting that DNA methylation hypermethylation of tumor suppressor exists in CTCs. Our results added a new dimension to the dissemination and metastasis. Whether the DNA methylation change in CTCs is of prognostic, predictive, or therapeutic importance has yet to be determined. 
EMT plasticity of CTCs reveals that epigenetic landscape is implicated in the dynamic events during dissemination and metastasis. The epithelial genes tend to be hypermethylated in their promoters, such as $C d h 1$, a key feature of EMT transition [33]. Such promoter hypermethylation leads to the loss of the epithelial features, and acquisition of a mesenchymal-like phenotype. As for the mesenchymal genes, the DNA methylation status showed plastic, which suggested that CTCs appear arrested in a biphenotypic state. Another observation suggests that CTCs have several properties that commonly feature stem cell implication. For instance, CTCs result in hypomethylation of TFBS of master stemness and proliferation regulators, such as CNOT3, KDM2B, and KLF10. Moreover, putative stem cell genes are hypomethylated in their promoters in CTCs, such as ALDH1A1 and CD44, suggesting that CTCs are endowed with a stemness-related feature. This finding reinforced the stem cell-like characteristics of CTCs in lung cancer.

Our LCM- $\mu$ WGBS method has several advantages. First, it is an efficient workflow, which can accurately and reproducibly profile the DNA methylation of microdissected CTC samples, and will facilitate the investigation of CTC DNA methylome. Second, this method is independent of loss of EpCAM expression. Classical CTC staining criteria include the presence of cell surface EpCAM. However, EpCAM is not a perfect marker for CTC selection due to the high variation in its gene expression. Third, we provided the CTC DNA methylome at single base resolution, which can discover a unique "CTC DNA methylation signature" that is distinct from primary tumors. Several limitations of our work should be addressed. First, only few lung cancer patients (15 patients) were involved in this analysis. Second, CTCs are very heterogeneous tumor cells; however, this method is difficult to attain single-cell resolution with the current LCM technology because many harvested cells do not remain intact after laser microdissection. Third, the field of single-cell epigenomics is still in its infancy.

Taken together, we provided a robust and efficient method for CTC DNA methylation analysis. Using this method, we revealed a detailed description of the DNA methylation landscape in lung cancer CTCs at single base-pair resolution. It might facilitate to propose noninvasive CTC DNA methylation biomarkers contributing to clinical diagnosis.

\section{Materials and methods}

\section{Patient specimens and cell lines}

Blood, primary tumors, and paired adjacent normal tissues were collected from lung cancer patients with written consent, which was approval by the Affiliated Hospital of
Xuzhou Medical University Agency Ethics Committee. We collected patient peripheral venous blood $(7.5 \mathrm{ml})$ using EDTA tubes and stored them at room temperature for processing within $24 \mathrm{~h}$. Tissue samples were snap-frozen in liquid nitrogen and stored at $-80^{\circ} \mathrm{C}$. Human lung cancer cell line A549 was purchased from Cell Bank of Type Culture Collection of the Chinese Academy of Sciences (Shanghai, China) and maintained in a humidified incubator at $37^{\circ} \mathrm{C}$, using minimum essential media, supplemented with fetal bovine serum $(10 \% \mathrm{v} / \mathrm{v})$.

\section{CTCs enrichment and detection}

An EpCAM-independent method was employed for CTCs subtraction enrichment. At first, red blood cells were lysed using RBC lysis buffer (G-bioscience) according to the manufacturer's protocol. The sample was then centrifuged at $800 \times g$ for $10 \mathrm{~min}$ at room temperature, and the supernatants above the red blood cells were removed to deplete serum. All the sedimented cells were mixed with $3 \mathrm{ml}$ nonhematopoietic cell separation matrix, followed by centrifugation at $400 \times g$ for $8 \mathrm{~min}$. White buffy was collected and subjected to magnetic separation of beads to deplete red blood cells. The resulting pellet containing rare cells was incubated with $150 \mu \mathrm{l}$ immunomagnetic particles coupled to anti-CD45 monoclonal antibody for $10 \mathrm{~min}$, followed by magnetic separation. The cell pellet was mixed with $100 \mu \mathrm{l}$ cell fixative solution and applied to the formatted and coated CTC slide. After the drying process, the slides were suitable for iFISH.

CTCs were detected by immunostaining of CD45, 4',6diamidino-2-phenylindole, dihydrochloride (DAPI), and FISH of the centromere of chromosome 8 probe (CEP8). CTCs are identified by combining immunofluorescent staining of CD45 and FISH with the CEP8 probe method. In brief, we added hybridization solution containing CEP8 probe to the slides. After hybridization, the antibody of CD45 was added to the slides. Finally, the nuclear dye DAPI (100 mg/ml, Sigma) was added and slides were mounted for microscopic observation. Cells with the DAPI+/CD45-/ CEP8 $\geq 3$ pattern were considered to be CTCs. Cells with $\mathrm{DAPI}+/ \mathrm{CD} 45+/ \mathrm{CEP} 8=2$ pattern were defined as white blood cells and cells with DAPI+/CD45-/CEP8 $=2$ pattern were defined as indeterminate cells.

\section{CTCs extraction using LCM}

The target cells in fixed slide were laser microdissected following the manufacturer's protocol for the PALM Laser MicroBeam System (Zeiss AG, Oberkochen, Germany). To facilitate LCM extraction, a PEN membrane-coated glass slide was used to prepare samples, which made it easily to be cut together with the samples. When we identified the 
target cell, a circle was drawn around it. Then, the area of the interest was laser microdissected, and catapulted into an adhesive cap of the collection tube. All CTCs harvested from each patient were pooled into one tube for subsequent $\mu \mathrm{WGBS}$ analysis.

\section{$\mu W G B S$ analysis}

The tumor tissues had been stained with $\mathrm{H} \& \mathrm{E}$ before $\mu$ WGBS to maximize sample tumor purity and the efficiency of tissue utilization. $\mu$ WGBS was performed according to the protocol of previous single-cell bisulfite sequencing echnology [7]. The PBAT method was used to avoid high DNA loss from limited starting material. Bisulfite conversion was performed according to the manufacturer's instructions for EZ DNA Methylation Direct Kit (Zymo Research D5020), and bisulfite-treated DNA was eluted using $9 \mu \mathrm{l}$ of elution buffer. Here, bisulfite treatment was performed directly on lysed cells by placing cells in $10 \mu \mathrm{l}$ digestion buffer and $1 \mu \mathrm{l}$ proteinase $\mathrm{K}$ for $20 \mathrm{~min}$ at $50{ }^{\circ} \mathrm{C}$. Sequencing library was prepared using the TruSeq DNA Methylation Kit (Illumina, EGMK91396) according to manufacturer's instructions. For library amplification, 18 PCR cycles were performed. The final library purification was performed twice using Agencourt AMPure XP beads. Library concentration was estimated using Qubit dsDNA HS Assay Kit. Sequencing was performed on Illumina HiSeq X Ten platform.

\section{Bioinformatics analysis of DNA methylation sequencing data}

Several bioinformatics steps were carried out to analyze the bisulfite sequencing reads: (1) adapter trimming, (2) alignment of bisulfite-treated reads to human genome, (3) determination of methylation state at each cytosine, and (4) filtering the contaminating reads. Library adapter was trimmed using trimmomatic [34], and fastqc is used to evaluate clean data. Bisulfite sequencing reads alignment was done using Bismark [35] with default parameters. All analyses were performed based on the human reference genome assembly hg38.

DMRs were determined using DMRseq [36] and bsseq software package [37]. In brief, we processed $\mathrm{CpG}$ count matrixes to merge symmetric $\mathrm{CpG}$ sites across stands and filtered those $\mathrm{CpG}$ sites for at least $1 \times$ coverage across the human genome, which accords with the minimum requirements for DMR inference. DMRs were identified by testing for differences between samples. We set the sliding window size and step at 1000 and $100 \mathrm{bp}$. DMRseq was employed by setting a cutoff of 0.05 and increasing the number of permutations to 500. Background regions were considered as the testable regions, and used as the candidate regions for permutation analysis to determine significant DMRs.

\section{TFBSs enrichment analysis in DMRs}

TFBS enrichment in DMRs was calculated using i-cisTarget software [26] (https://gbiomed.kuleuven.be/apps/lcb/i-cisTa rget/) based on the normalized enrichment score (NES). NES corresponds to the enrichment score, which reflects the degree to which the motif set is overrepresented at the top or bottom of a ranked list of motifs. NES is positive if it was enriched in DMRs. NES score threshold $>3.4$ was used (passes a FDR threshold of 0.05). Motif search is performed in DMR regions overlapping with predefined candidate regularity regions.

\section{Functional enrichment analysis}

Functional enrichment analysis of KEGG was performed using the using the DAVID software [38] for the methylation related genes. The KEGG categories were corrected using the Benjamini-Hochberg method, and the genes with F.D.R of less than 0.05 were considered to be significantly enriched.

\section{Data availability}

The bisulfite sequencing data have been deposited in the NCBI Sequencing Read Archive database (SRA, http://www.ncbi.nlm.nih.gov/sra/) under the accession number PRJNA649023.

Funding This work was supported by grants from Xuzhou Medical University (grant D2019027), Natural Science Fund for Colleges and Universities in Jiangsu Province (20KJA320007), and the Training Project for Youth Teams of Science and Technology Innovation at Xuzhou Medical University (TD202008).

Author contributions DD designed the study and wrote the manuscript. All authors carried out the data analysis and read and approved the final manuscript.

\section{Compliance with ethical standards}

Conflict of interest The authors declare that they have no conflict of interest.

Consent to participate All patients have given written informed consent prior to the inclusion in the project.

Ethical approval Institutional Review Board approved the study.

Publisher's note Springer Nature remains neutral with regard to jurisdictional claims in published maps and institutional affiliations. 
Open Access This article is licensed under a Creative Commons Attribution 4.0 International License, which permits use, sharing, adaptation, distribution and reproduction in any medium or format, as long as you give appropriate credit to the original author(s) and the source, provide a link to the Creative Commons license, and indicate if changes were made. The images or other third party material in this article are included in the article's Creative Commons license, unless indicated otherwise in a credit line to the material. If material is not included in the article's Creative Commons license and your intended use is not permitted by statutory regulation or exceeds the permitted use, you will need to obtain permission directly from the copyright holder. To view a copy of this license, visit http://creativecommons. org/licenses/by/4.0/.

\section{References}

1. Pantel K, Speicher MR. The biology of circulating tumor cells. Oncogene. 2016;35:1216-24.

2. Faugeroux V, Lefebvre C, Pailler E, Pierron V, Marcaillou C, Tourlet $\mathrm{S}$, et al. An accessible and unique insight into metastasis mutational content through whole-exome sequencing of circulating tumor cells in metastatic prostate cancer. Eur Urol Oncol. 2020;3:498-508.

3. Miyamoto DT, Zheng Y, Wittner BS, Lee RJ, Zhu H, Broderick KT, et al. RNA-seq of single prostate CTCs implicates noncanonical Wnt signaling in antiandrogen resistance. Science. 2015;349:1351-6.

4. Yu M, Ting DT, Stott SL, Wittner BS, Ozsolak F, Paul S, et al. RNA sequencing of pancreatic circulating tumour cells implicates WNT signalling in metastasis. Nature. 2012;487:510-3.

5. Boral D, Vishnoi M, Liu HN, Yin W, Sprouse ML, Scamardo A, et al. Molecular characterization of breast cancer CTCs associated with brain metastasis. Nat Commun. 2017;8:196.

6. Allard WJ, Matera J, Miller MC, Repollet M, Connelly MC, Rao $\mathrm{C}$, et al. Tumor cells circulate in the peripheral blood of all major carcinomas but not in healthy subjects or patients with nonmalignant diseases. Clin Cancer Res. 2004;10:6897-904.

7. Farlik M, Sheffield NC, Nuzzo A, Datlinger P, Schonegger A, Klughammer J, et al. Single-cell DNA methylome sequencing and bioinformatic inference of epigenomic cell-state dynamics. Cell Rep. 2015;10:1386-97.

8. Wu S, Liu S, Liu Z, Huang J, Pu X, Li J, et al. Classification of circulating tumor cells by epithelial-mesenchymal transition markers. PLoS ONE. 2015;10:e0123976.

9. Yu M, Bardia A, Wittner BS, Stott SL, Smas ME, Ting DT, et al. Circulating breast tumor cells exhibit dynamic changes in epithelial and mesenchymal composition. Science. 2013;339:580-4.

10. Park ES, Yan JP, Ang RA, Lee JH, Deng X, Duffy SP, et al. Isolation and genome sequencing of individual circulating tumor cells using hydrogel encapsulation and laser capture microdissection. Lab Chip. 2018;18:1736-49.

11. Jones PA. Functions of DNA methylation: islands, start sites, gene bodies and beyond. Nat Rev Genet. 2012;13:484-92.

12. Ziller MJ, Gu H, Muller F, Donaghey J, Tsai LT, Kohlbacher O, et al. Charting a dynamic DNA methylation landscape of the human genome. Nature. 2013;500:477-81.

13. Hansen KD, Timp W, Bravo HC, Sabunciyan S, Langmead B, $\mathrm{McDonald} \mathrm{OG}$, et al. Increased methylation variation in epigenetic domains across cancer types. Nat Genet. 2011;43:768-75.

14. Hon GC, Hawkins RD, Caballero OL, Lo C, Lister R, Pelizzola $\mathrm{M}$, et al. Global DNA hypomethylation coupled to repressive chromatin domain formation and gene silencing in breast cancer. Genome Res. 2012;22:246-58.

15. Jones PA, Baylin SB. The epigenomics of cancer. Cell. 2007;128:683-92.

16. Berman BP, Weisenberger DJ, Aman JF, Hinoue T, Ramjan Z, Liu Y, et al. Regions of focal DNA hypermethylation and long-range hypomethylation in colorectal cancer coincide with nuclear lamina-associated domains. Nat Genet. 2011;44:40-46.

17. Vidal E, Sayols S, Moran S, Guillaumet-Adkins A, Schroeder MP, Royo R, et al. A DNA methylation map of human cancer at single base-pair resolution. Oncogene. 2017;36:5648-57.

18. Chimonidou M, Strati A, Tzitzira A, Sotiropoulou G, Malamos N, Georgoulias V, et al. DNA methylation of tumor suppressor and metastasis suppressor genes in circulating tumor cells. Clin Chem. 2011;57:1169-77.

19. Chimonidou M, Kallergi G, Georgoulias V, Welch DR, Lianidou ES. Breast cancer metastasis suppressor-1 promoter methylation in primary breast tumors and corresponding circulating tumor cells. Mol Cancer Res. 2013;11:1248-57.

20. Chimonidou M, Strati A, Malamos N, Georgoulias V, Lianidou ES. SOX17 promoter methylation in circulating tumor cells and matched cell-free DNA isolated from plasma of patients with breast cancer. Clin Chem. 2013;59:270-9.

21. Gkountela S, Castro-Giner F, Szczerba BM, Vetter M, Landin J, Scherrer R, et al. Circulating tumor cell clustering shapes DNA methylation to enable metastasis seeding. Cell. 2019;176:98-112. e114.

22. Pixberg CF, Raba K, Muller F, Behrens B, Honisch E, Niederacher D, et al. Analysis of DNA methylation in single circulating tumor cells. Oncogene. 2017;36:3223-31.

23. Liu X, Zhang Z, Zhang B, Zheng Y, Zheng C, Liu B, et al. Circulating tumor cells detection in neuroblastoma patients by EpCAM-independent enrichment and immunostaining-fluorescence in situ hybridization. EBioMedicine. 2018;35:244-50.

24. Zhang Y, Wang F, Ning N, Chen Q, Yang Z, Guo Y, et al. Patterns of circulating tumor cells identified by CEP8, $\mathrm{CK}$ and $\mathrm{CD} 45$ in pancreatic cancer. Int $\mathrm{J}$ Cancer. 2015;136:1228-33.

25. Zheng X, Zhao Q, Wu HJ, Li W, Wang H, Meyer CA, et al. MethylPurify: tumor purity deconvolution and differential methylation detection from single tumor DNA methylomes. Genome Biol. 2014;15:419.

26. Herrmann C, Van de Sande B, Potier D, Aerts S. i-cisTarget: an integrative genomics method for the prediction of regulatory features and cis-regulatory modules. Nucleic Acids Res. 2012;40:e114.

27. Baron V, Adamson ED, Calogero A, Ragona G, Mercola D. The transcription factor Egr1 is a direct regulator of multiple tumor suppressors including TGFbeta1, PTEN, p53, and fibronectin. Cancer Gene Ther. 2006;13:115-24.

28. Lee CC, Chen WS, Chen CC, Chen LL, Lin YS, Fan CS, et al. TCF12 protein functions as transcriptional repressor of E-cadherin, and its overexpression is correlated with metastasis of colorectal cancer. J Biol Chem. 2012;287:2798-809.

29. Engelmann D, Putzer BM. The dark side of E2F1: in transit beyond apoptosis. Cancer Res. 2012;72:571-5.

30. Lamouille S, Xu J, Derynck R. Molecular mechanisms of epithelial-mesenchymal transition. Nat Rev Mol Cell Biol. 2014;15:178-96.

31. Keller L, Pantel K. Unravelling tumour heterogeneity by singlecell profiling of circulating tumour cells. Nat Rev Cancer. 2019;19:553-67.

32. Zheng G, Ma Y, Zou Y, Yin A, Li W, Dong D. HCMDB: the human cancer metastasis database. Nucleic Acids Res. 2018;46: D950-5.

33. Grady WM, Willis J, Guilford PJ, Dunbier AK, Toro TT, Lynch $\mathrm{H}$, et al. Methylation of the $\mathrm{CDH} 1$ promoter as the second genetic hit in hereditary diffuse gastric cancer. Nat Genet. 2000;26:16-17.

34. Bolger AM, Lohse M, Usadel B. Trimmomatic: a flexible trimmer for Illumina sequence data. Bioinformatics. 2014;30:2114-20. 
35. Krueger F, Andrews SR. Bismark: a flexible aligner and methylation caller for Bisulfite-Seq applications. Bioinformatics. 2011;27:1571-2.

36. Korthauer K, Chakraborty S, Benjamini Y, Irizarry RA. Detection and accurate false discovery rate control of differentially methylated regions from whole genome bisulfite sequencing. Biostatistics. 2019;20:367-83.
37. Hansen KD, Langmead B, Irizarry RA. BSmooth: from whole genome bisulfite sequencing reads to differentially methylated regions. Genome Biol. 2012;13:R83.

38. Huang DW, Sherman BT, Tan Q, Collins JR, Alvord WG, Roayaei J, et al. The DAVID gene functional classification tool: a novel biological module-centric algorithm to functionally analyze large gene lists. Genome Biol. 2007;8:R183. 